\title{
An Efficient Face Recognition with ANN using Hybrid Feature Extraction Methods
}

\author{
Mithila Sompura \\ Student of M.E in Computer Engineering \\ Department, Hashmukh Goswami College of \\ Engineering and Technology \\ Ahmedabad, India
}

\author{
Vinit Gupta \\ Assistant Professor in Computer Engineering \\ Department, Hashmukh Goswami College of \\ Engineering and Technology \\ Ahmedabad, India
}

\begin{abstract}
Now a day's face recognition is the most interesting and active research area in the field of phycology, neuroscience, and computer vision. In this paper a fast efficient algorithm is developed with the better recognition rate of face in different conditions that is illumination, head pose, expressions etc. In which we have extract the global and local features using PCA (Principle Component Analysis) and LBP (Local Binary Pattern) respectively. we have experiment the proposed algorithm with the standard Yale database which contains 15 individuals with each contains 11 images $\left(15^{*} 11\right)$. So the fusion of Global and Local features are fed to the MLP (Multilayer Perceptron). The BPMLP (Backpropagation Multilayer Perceptron) is used for the classification. The proposed method achieves $93 \%$ accuracy compare to the existing approach.
\end{abstract}

\section{Keywords}

PCA (Principle Component Analysis), LDA (Linear Discriminant Analysis), LBP (Local Binary Pattern), BPMLP (Backpropagation Multilayer perceptron), k-NN (k- nearest neighbor), ICA (Independent Component Analysis), KSOM (Kohonen Self organizing Map), LPP (Linear Parallel Projection)

\section{INTRODUCTION}

Biometric identification has some limitation with respect to advancement in today's era. The limitation of Biometric contains the misplaced of passwords or pins, may fingure get corrupted or damage etc. Many companies use the biometric for the daily attendance of the employee which sometimes not work properly even multiple password are require for logging to each individual system. So the face recognition has the advantage compare to Biometric Technique.

Now a day's face recognition is the more interesting and active research area but it still has some limitation that is to recognize the individual person in any environment is the most challenging task [1]. The most popular global feature extraction methods are PCA [2], LDA [3] and ICA. The face Recognition system generally divided in to three categories: 1) Preprocessing: these stages normalize and eliminate the background noise which every face normally contains. 2) Feature Extraction: this stage divided in to two stages that is feature based and holistic based. Feature based method considers the individual feature of the face that is eyes, nose, mouth, chin etc. and find the position relationship between each features. Whereas in holistic based method whole face is analyzed as the image. Holistic based method is generally implemented for the feature vector because the facial features are sometimes difficult to detect with the different rotation, variation and scale. 3) Classification: in this
Stage the powerful classifiers are used according to the requirement of the application. To reduce the error rate in the image the author [4] have used the PCA and LDA with the modified methods with the uses of subspace techniques.

By combining the global and local feature using analytic and holistic approach the author [5] have achieved 97\% of recognition rate using artificial neural network.

In paper [6] author have used Gabor Wavelet Filter to extract the Local and Global features and experimented on k-NN and multi class SVM classifier. In [7] to preserve the local structure of the face author have used LPP (Locality Preserving Projection) but as a result LPP is failed to detect the variation of the face which is nonlinear so that RBFN (Radial Basis Function Network) is used for the improve the performance. In paper [8] to recognize the expression of the face author have used KSOM (Kohonen Self Organizing Map) which cluster the data in to smaller zones to keeps the topology of the input vector and from equally subdivided block features are extracted using LBP (Local Binary Pattern). For the correct face identification with rotated and location invariant face the author have used Backpropagation algorithm [9]. In paper [10] author have developed and experimented on conventional PCA (Principle Component Analysis) and 2DPCA (Two-dimensional PCA). In which author have used k-NN for the recognition task. In [11] author have tested the different algorithms on R1 face database in which they have proved that LBP can meet the requirement of both the accuracy in rate and time if there are sufficient samples in each images in variety of environment situations. In [12] author have used SLGS (Symmetric Local Graph Structure) based on the LGS (Local Graph Structure) in which each pixel is defined with the graph structure of its neighbor's pixel. For the recognition they have used the nearest neighbor classifier with tested on Yale and AT\&T face database [18].

In this paper we have only give attention on feature extraction and classification rather on normalization and face detection. We have used the fundamentals of fusion of both Global and Local features with the use of PCA (Principle Component Analysis) and LBP (Local Binary Pattern) respectively. As discussed in the literature review PCA is used to extract the global features and LBP is used to extract local features by dividing the whole image in to $8 * 8$ blocks. Fusion of both the methods result in to one vector and that vector is used by the MLP (Multilayer Perceptron). For the better classification we have used BPMNLP (Backpropagation Multilayer Perceptron) classifier which outperformed the other existing methods.

The organization of the paper is as follows: In section II Theory of operation with different methods. In section III Proposed work. In section IV the experiment and result are tested using Yale face database with performance ration and 
finally we have presented our work with the conclusion in section $\mathrm{V}$.

\section{THEORY OF OPERATION}

\subsection{Principle Component Analysis}

Principal component analysis (PCA) is a mathematical procedure that uses to convert a set of observations of possibly correlated variables into a set of values of uncorrelated variables called principal components [4] and it is referred to as linear method [17].Main idea of PCA (Karhunen-Loeve expansion) [10] for faces is to find vectors that best account for variation of face images in entire image space. In [6] author had used PCA to reduce the dimension of a fused image. This method is mainly used as dimension reduction which finds vectors and A group of Eigenfaces is extracted from the original image. These vectors are called Eigen-vectors. Linear combination obtained using Principle component are called Eigenface [10]. Compute the covariance matrix M [14]

$$
\mathrm{M}=\frac{1}{n} \sum_{i=1}^{n}\left(x_{i}-\mu\right)\left(x_{i}-\mu\right)^{\mathrm{T}}
$$

\subsection{Linear Discriminant Analysis}

LDA is an enhancement to PCA Constructs a discriminant subspace that minimizes the scatter between images of same class and maximizes the scatter between different class images [15]. The between-class scatter matrix $V_{b}$, within-class scatter matrix $\mathrm{V}_{\mathrm{w}}$ and the projective matrix $\mathrm{P}$ are defined as follows [7]:

$$
\mathrm{V}_{\mathrm{w}}=\sum_{i=1}^{\mathrm{c}}\left(\sum_{j=1}^{N_{i}}\left(x_{j}^{(i)}-m_{i}\right)\left(x_{j}^{(i)}-m_{i}\right)^{T}\right)
$$

$$
\mathrm{V}_{\mathrm{b}}=\sum_{i=1}^{\mathrm{c}} N_{i}\left(m_{i}-m\right)\left(m_{i}-m\right)^{T}
$$

$$
\mathrm{P}=\arg \max \left|\frac{\mathrm{P}^{\mathrm{T}} \mathrm{V}_{\mathrm{b}} \mathrm{P}}{\mathrm{P}^{\mathrm{T}} \mathrm{V}_{\mathrm{w}} \mathrm{P}}\right|
$$

Where $m_{i}$ and $N_{i}$ are the mean face and sample number in individual face class respectively, $x_{j}^{(i)}$ is the $\mathrm{j}$-th sample in the $i^{\text {th }}$ class. For recognition, the linear distance function is computed as:

$\mathrm{d}_{\mathrm{LDA}}=W^{T}(T-P)$

While taking class discriminatory information at that time perform dimensionality reduction. When classes are separated seek to find direction. Due to variation in illumination and expression LDA is more capable of distinguishing image variation.

\subsection{Local Binary Pattern}

This is the most powerful method for the face recognition which describes the image as a texture and it is mainly divided in to three different features which are: pixel level, region level and global level [11]. This method assigns the label to each pixel by the $3 * 3$ neighborhood of each pixel and assigns the one pixel as a center pixel with the threshold and that label of histogram is used as texture. LBP is most widely used in face recognition because of its unique feature and less computation time [12]. Fig gives an example of LBP operator

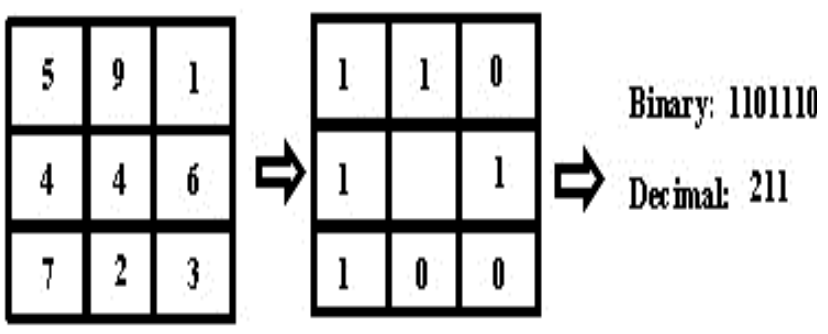

Convert to Thresholl

Fig 1: Example of LBP operation

The disadvantage of the LBP operator is that if there is the large structure with the dominant features the $3 * 3$ neighborhood is too small. So later on this limitation is overcome by extending the neighborhood with different sizes. [19]. As shown in the above pattern is uniform, so we can say LBP is uniform if contains the combination of 0 and 1 when seen as a circular string. For example 00000000, 11010010 are uniform patterns. For the face representation the whole face $f$ is divided in to small regions say $r 1, r 2 \ldots . r f$. The histogram of all the local regions are taken and combined together from that the similar histograms are taken for the face representation.

\subsection{Multi-layer feed forward}

Multilayer Feed-Forward network consists of multiple layers. In [5] they have used Multi-layer feed forward network which consists of multiple layers to increase the recognition performance rate. This architecture having input and output layer with one or more intermediary layers called hidden layer which are also known as Hidden Neurons. Before direction input to the hidden layer intermediate computation are carried out at the hidden layer.

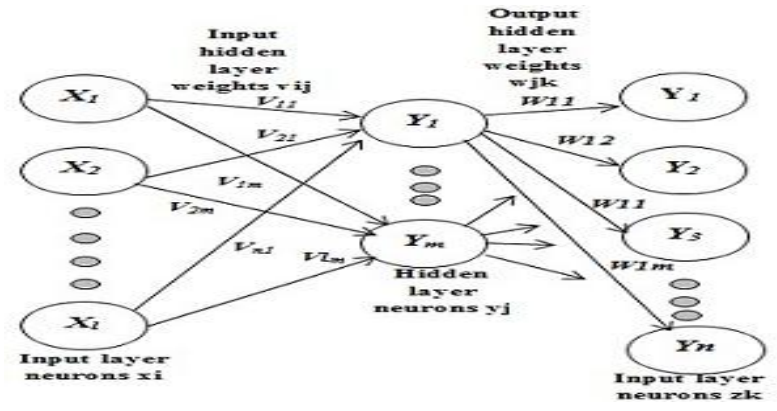

Fig 2: Multi-layer feed forward network [5]. 


\section{PROPOSED WORK}

In this section of proposed algorithm with the flowchart is discussed. To overcome the limitation of feature based method we have first apply the Principle component analysis (PCA) to extract the global features considering whole face. As discussed we know that every face varies with different pose, invariants, illumination etc. therefore rather only considering whole face we have also considered the local features by dividing the whole face in to sub-blocks using the Local Binary Pattern (LBP).

The proposed algorithm is summarized as follows:

1) Extraction of Global features: principle component analysis method is applied on whole image say $I$. Get most significant Eigenfaces from $I$ and store in vector $S$.

2) Extraction of Local features: local features are extracted using Local binary pattern method and store the features in to $S 1, S 2, S 3$, and $S 4$.

3) Fusion of Local and Global features: In this step the fusion of both local and global features are combined extracted from step1 and step2. The new feature vector is created say:

$$
F=(S, S 1, S 2, S 3)^{T}
$$

4) Classification: for the correct classification of the face we have used BPMLP.

The working of our proposed algorithm is described in Figure 7.

\section{EXPERIMENTS AND RESULTS}

We have made our system to be able to learn under this configuration: Intel Core 2(I3)-3110M CPU @ 2.40GHZ with 64 bit operating system. In this paper we have tested our proposed algorithm with YALE Face Database which contains 165 grayscale images $\left(15^{*} 11\right)$. There are 15 individuals where each has 11 pictures with the size of $50 * 50$. We have cropped those images in to $32 * 32$ dimensions (height and width). Each individual contains images having different facial expressions, and with or without glasses. For our system we have taken the 10 images for training sample and 1 as testing sample by considering the training images should not be same as a testing image. For each experiments we have consider all the parameters which are varied with different parameter comparison such as number of epochs, accuracy, training time by comparing with $\mathrm{CPU}$ weight range between $-1,1$ and $-0.5,0.5$.

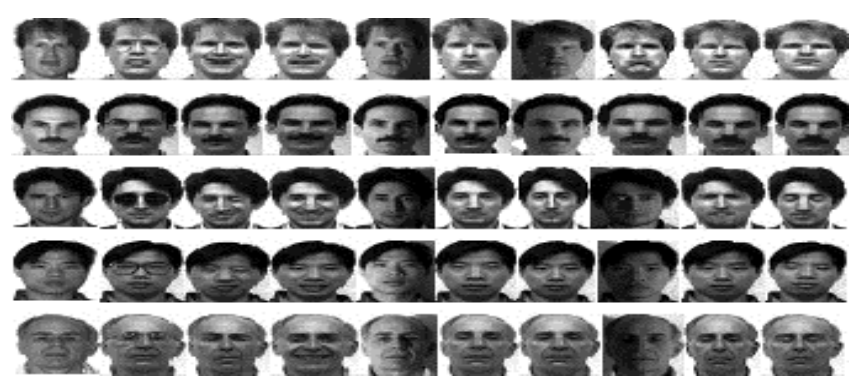

Fig 3: Example of face images taken from YALE face database.

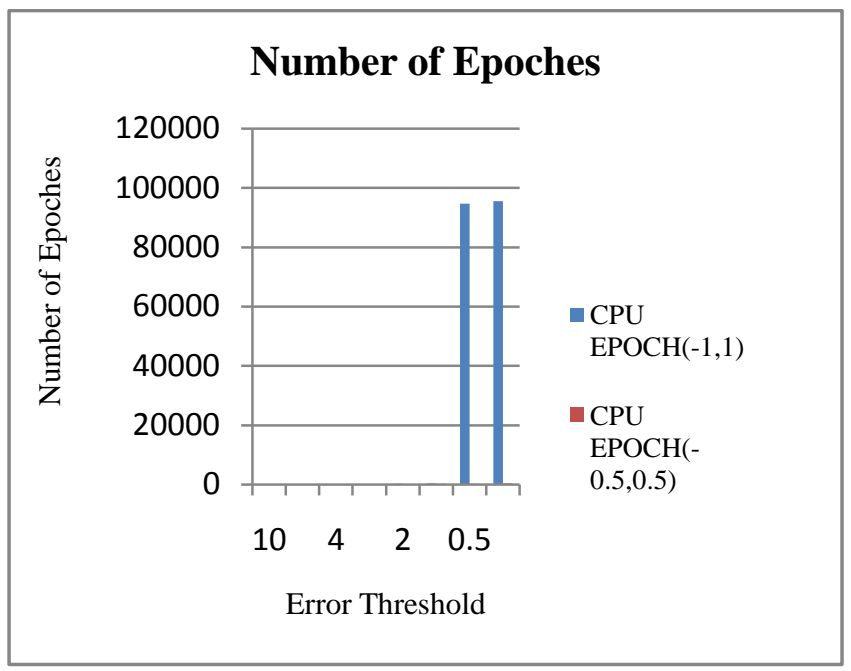

Fig 4: Number of Epochs

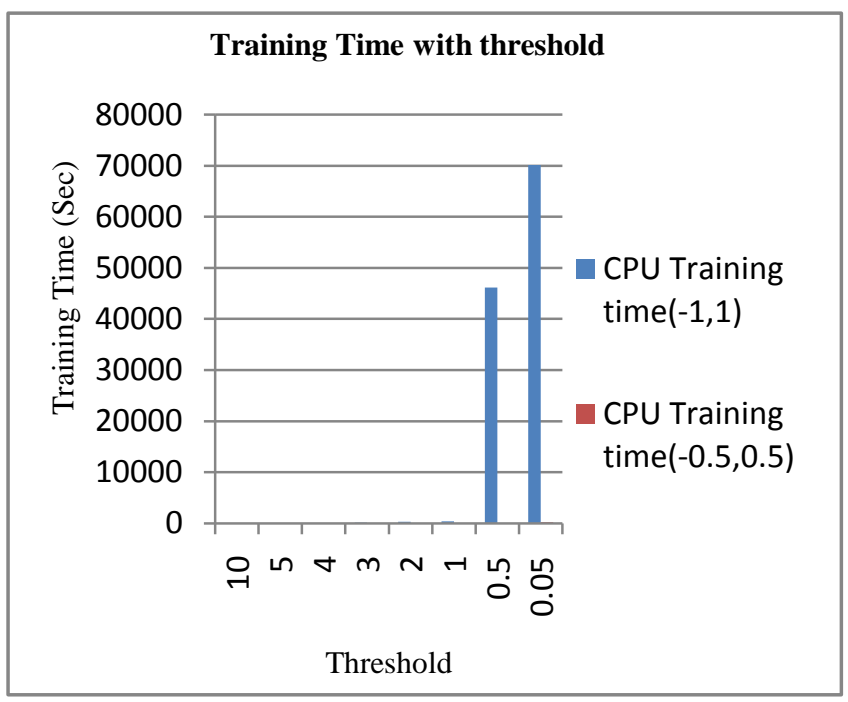

Fig 5: Training time by comparing different threshold

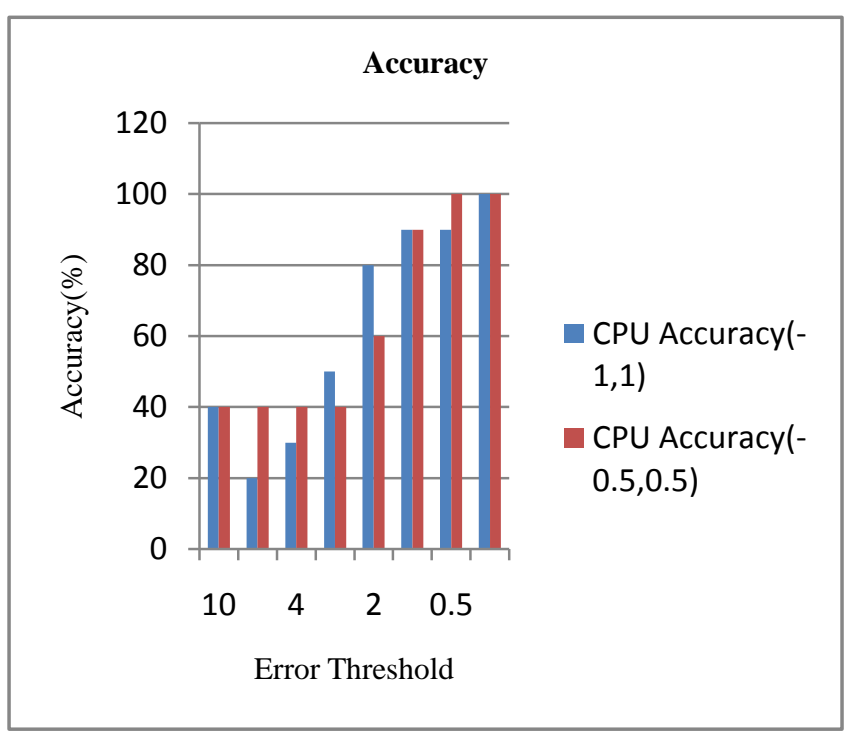

Fig 6: Accuracy of the system 
Table 1. Comparison of accuracy with respect to Error Threshold

\begin{tabular}{|c|c|c|}
\hline $\begin{array}{c}\text { Error } \\
\text { Threshold }\end{array}$ & $\begin{array}{c}\text { CPU Accuracy } \\
(\mathbf{- 1 , 1 )}\end{array}$ & $\begin{array}{c}\text { CPU Accuracy } \\
(\mathbf{- 0 . 5 , 0 . 5 )}\end{array}$ \\
\hline 10 & 40 & 40 \\
\hline 5 & 20 & 40 \\
\hline 4 & 30 & 40 \\
\hline 3 & 50 & 60 \\
\hline 2 & 80 & 90 \\
\hline 1 & 90 & 100 \\
\hline 0.5 & 90 & \\
\hline 0.05 & 100 & \\
\hline
\end{tabular}

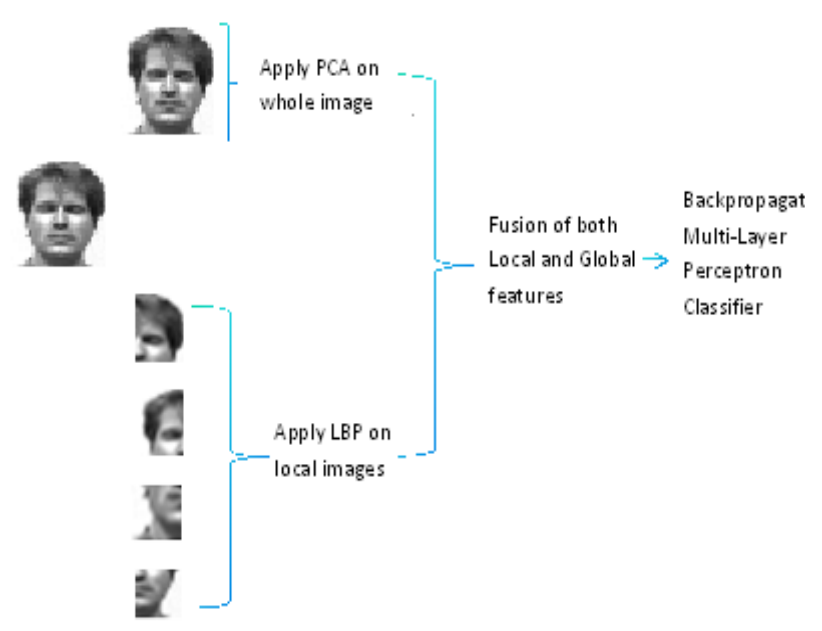

Fig 7: Working of Proposed system

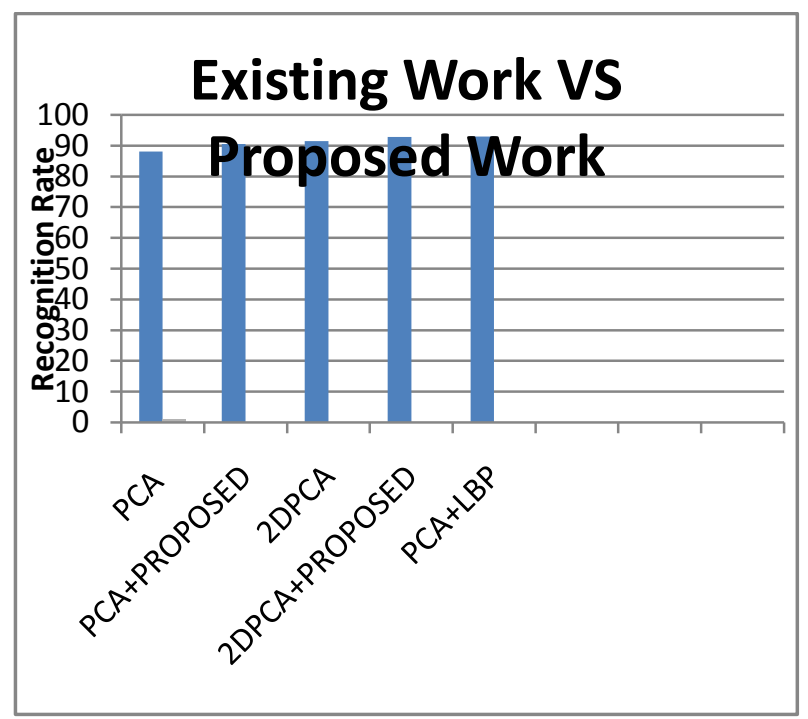

Fig 8: Comparison of Existing work [10] with proposed work
Table 2. Comparison of Training time with respect Error threshold

\begin{tabular}{|c|c|c|}
\hline $\begin{array}{c}\text { Error } \\
\text { Threshold }\end{array}$ & $\begin{array}{c}\text { CPU Training time } \\
(\mathbf{- 1 , 1 )}\end{array}$ & $\begin{array}{c}\text { CPU Training } \\
\text { time(-0.5,0.5) }\end{array}$ \\
\hline 10 & 11.824 & 17.9027 \\
\hline 5 & 31.2471 & 14.4381 \\
\hline 4 & 54.2085 & 13.167 \\
\hline 3 & 180.89582 & 31.661 \\
\hline 2 & 309.22135 & 59.8935 \\
\hline 1 & 413.2058 & 107.4457 \\
\hline 0.5 & 46156.192 & 118.5458 \\
\hline 0.05 & 70164.0603 & 220.922 \\
\hline
\end{tabular}

Table 3. Comparison Table

\begin{tabular}{|c|c|c|}
\hline Strategy & Method & $\begin{array}{c}\text { Recognition } \\
\text { Rate }(\boldsymbol{\%})\end{array}$ \\
\hline \multirow{3}{*}{$\begin{array}{c}\text { Five images as } \\
\text { Training } \\
\text { (Existing } \\
\text { approach) }\end{array}$} & PCA & 88.1 \\
\cline { 2 - 3 } & PCA+PROPOSED & 90.5 \\
\cline { 2 - 3 } & 2DPCA+PROPOSED & 91.5 \\
\hline \multirow{2}{*}{$\begin{array}{c}\text { Nine images as } \\
\text { Training } \\
\text { (Proposed } \\
\text { approach) }\end{array}$} & PCA+LBP & \\
& & \\
& & \\
\hline
\end{tabular}

\section{PERFORMANCE OF OUR SYSTEM}

Our system performs better than the earlier methods and identifies $100 \%$ accuracy with both weight range $(-1,1)$ and $(-$ $0.5,0.5)$. But as our experiment when we decrease the threshold value nearer to 0.5 it takes much training time but recognize the person correctly in any environment. So as shown in the figure 5 if we set the weight range to $(-1,1)$ the system takes less time to train the network and give the same result as compare to $(-0.5,0.5)$.

\section{CONCLUSION}

Face recognition is the core problem in computer vision because of highly nonlinearity which is widely applicable in many domains. In this paper we have extract the Global and Local features using PCA and LBP respectively. The fusion of both the Global and Local features are fed to the MLP (Multilayer Perceptron). To increase the performance of our system in terms of recognition rate and training time we have used the BP-MLP (Back propagation multilayer perceptron). 
As result we found that our system performs well in any condition illumination, pose, light etc.

This work can be extended by recognizing the hundreds of image with each image contains different expression so at that instance the system should take less time for the classification with better performance rate.

\section{REFERENCES}

[1] Rabia Jafri and Hamid R. Arabnia, "A Survey of Face Recognition Techniques, Journal of Information Processing Systems", Vol.5, No.2, June 2009 DOI : 10.3745/JIPS.2009.5.2.04

[2] M A Turk, and A P Pentland, "Face recognition using eigenfaces", Proc. IEEE Conference on Computer Vision and Pattern Recognition, 1991, pp. 586-591

[3] P N Belhumeur, J P Hespanha, and D J Kriegman," Eigenfaces vs. fisherfaces: Recognition using class specific linear projection", IEEE Transactions on Pattern Analysis and Machine Intelligence, Vol. 19, No.7, 1997, pp. 711-720.

[4] G. Prabhu Teja, S. Ravi," Face Recognition using Subspaces Techniques", 2012 IEEE

[5] Kolhandai Yesu, Himadri Jyoti Chakravorty, Prantik Bhuyan, Rifat Hussain, Kaustubh Bhattacharyya," Hybrid Features Based Face Recognition Method Using Artificial Neural Network", 2012 IEEE.

[6] Sara Nazari, Mohammad-Shahram Moin "Face Recognition Using Global and Local Gabor Features".

[7] Jian-qiang Mei, Zheng-guang Liu, Ming Ming," Application of Radial Basis Function Network and Locality Preserving Projections for Face Recognition", International Conference on Natural Computation, 2007 IEEE

[8] Anima Majumder_, Laxmidhar Behera and Venkatesh K. Subramanian, "Local Binary Pattern based Facial Expression Recognition using Self-organizing Map",
2014 International Joint Conference on Neural Networks (IJCNN) July 6-11, 2014, Beijing, China

[9] Dhananjoy Bhakta, Goutam Sarker, "A Rotation and Location Invariant Face Identification and Localization with or Without Occlusion using Modified RBFN",2013 IEEE.

[10] Swarup Kumar Dandpat and Prof. Suakadev Meher," Performance Improvement for Face Recognition Using PCA and Two-Dimensional PCA”, 2013 IEEE

[11] Bai Limin Jia Mingxing, Qiao Shengyang, Wu Qiang," A comparative study of Face Recognition Algorithms on R1 Face atabase",2014 IEEE.

[12] Mohd Fikri Azli Abdullah, Md Shohel Sayeed, Kalaiarasi Sonai Muthu, Housam Khalifa Bashier, Afizan Azman, Siti Zainab Ibrahim," Face recognition with Symmetric Local Graph Structure (SLGS)", Expert Systems with Applications 41 (2014) 6131-6137.

[13] J.Prabin Jose P.Poornima Kukkapalli Manoj Kumar," A Novel Method for Color Face Recognition Using KNN Classifier”, 2013 IEEE

[14] Philipp Wagner," Face Recognition with GNU Octave/MATLAB”, July 18, 2012.

[15] Ion Marqu'es," Face Recognition Algorithms", June 16, 2010

[16] Jiawei Han, Micheline Kamber, Jian Pei, "Data Mining Concepts and Techniques", 2012.

[17] Simon Haykin, "Neural Networks and Learning Machines", 2013

[18] Yale face Database, http://cvc.yale.edu/projects/yalefaces.

[19] T Ojala, M Pietik inen, and T M Enp, Multiresolution gray scale and rotation invariant texture analysis with local binary patterns, IEEE Transactions on Pattern Analysis and Machine Intelligence, Vol. 24, No. 7, 2002, pp. 971-987. 